\title{
SIP-based IMS Registration Analysis for WiMax-3G Interworking Architectures
}

\author{
Arslan Munir and Ann Gordon-Ross* \\ Department of Electrical and Computer Engineering \\ University of Florida, Gainesville, Florida, USA \\ e-mail: amunir@ufl.edu \& ann@hcs.ufl.edu \\ *Also with the NSF Center For High-Performance Reconfigurable Computing \\ at the University of Florida, USA
}

\begin{abstract}
The 3rd generation partnership project (3GPP) and $3 G P P 2$ have standardized the IP multimedia subsystem (IMS) to provide ubiquitous and access network independent IP-based services for next generation networks via merging cellular networks and the Internet. The application layer Session Initiation Protocol (SIP), standardized by $3 G P P$ and $3 G P P 2$ for IMS, is responsible for IMS session establishment, management, and transformation. The IEEE 802.16 worldwide interoperability for microwave access (WiMax) promises to provide high data rate broadband wireless access services. In this paper, we analyze the SIPbased IMS registration signaling delay for 3rd generation $(3 G)$ cellular and WiMax networks with specific reference to their interworking architectures. We also explore the effects of different WiMax-3G interworking architectures on the IMS registration signaling delay.
\end{abstract}

\section{Introduction}

The 3rd generation partnership project (3GPP) and 3GPP2 have standardized the IP multimedia subsystem (IMS) to provide IP-based rich multimedia services as well as content-based monetary charges for next generation networks. The application layer Session Initiation Protocol (SIP), standardized by $3 \mathrm{GPP}$ and $3 \mathrm{GPP} 2$ for IMS, is responsible for IMS session establishment, management, and transformation. The Internet Engineering Task Force (IETF) developed signaling compression (SigComp) for text-based protocol compression [12]. Even though the IEEE 802.16 worldwide interoperability for microwave access (WiMax) offers high data rates, WiMax coverage is limited when compared with $3 \mathrm{G}$ networks, which provide ubiquitous connectivity. On the other hand, $3 \mathrm{G}$ network data rates are much lower than WiMax data rates. The complementary coverage area and data rate characteristics of WiMax and 3G networks make their interworking, with the intent of ubiquitous high speed wireless data access, an interesting topic.

Two popular system models for interworking wireless access networks (ANs) (such as 3G with WiMax) are tight and loose coupling. In tightly coupled systems, the connecting AN integrates with the core $3 \mathrm{G}$ network similarly to any other $3 \mathrm{G}$ radio $\mathrm{AN}$, using the same authentication, mobility, and billing infrastructures. To communicate with the $3 \mathrm{G}$ network, the connecting AN implements $3 \mathrm{G}$ radio access network protocols to route traffic through core $3 \mathrm{G}$ elements. In loosely coupled systems, a connecting AN integrates into the core $3 \mathrm{G}$ network by routing communication traffic through the Internet, with no direct connection between the two networks. The two networks use different authentication, billing, and mobility protocols, however, they may share the same subscriber databases for customer record management. Tightly and loosely coupled architectures are proposed and their cost analyzed in [7] and [8], respectively. Also the interworking architectures that integrate 3G, WiMax, WLAN, and satellite ANs with IMS support are proposed and cost analyzed in [11].

An important aspect of interworking architectures is their IMS signaling efficiency, which is determined by the interworking architecture's ability to carry out the IMS signaling procedures (i.e., session establishment, registration, termination, and transformation) with minimum delay and overhead. Previous work provides limited IMS signaling delay analysis. [9] studied the IMS session establishment procedure when both the source node $(\mathrm{SN})$ and the correspondent node $(\mathrm{CN})$ are in CDMA2000. In this paper we consider a more general case where $\mathrm{SN}$ and $\mathrm{CN}$ are in different ANs. [5] studied SIP-based voice over IP (VoIP) IMS session establishment delay for $3 \mathrm{G}$ wireless networks using an adaptive retransmission timer for lost packet retransmission. The authors studied different protocols such as transmission control protocol (TCP), user datagram protocol (UDP), and radio link protocol (RLP). [10] studied 
SIP-based IMS session establishment for WiMax and 3G networks. However, this work did not analyze the IMS registration procedure and the effects of different interworking architectures on IMS signaling delay.

To the best of our knowledge, previous work only provides partial IMS signaling delay analysis. For example, the IMS registration DIAMETER authentication procedure has not been considered. Specifically, "reg event" (informs users of their registration status within the IMS network) was not considered. Furthermore, the majority of previous work isolates the IMS signaling procedures from the interworking architectures. The effects of this interaction are important because different interworking architectures contribute different delay and overhead to IMS signaling procedures.. Hence, IMS signaling delay analysis incorporating different interworking architectures evaluates the interworking architectures as well. Thus, the main contributions of this paper are:

- We analyze the SIP-based IMS registration signaling delay (including subscription to reg event state) for various $3 \mathrm{G}$ and WiMax channel rates using a comprehensive model, which considers transmission, processing, and queuing delays at each network node. Our analysis considers provisional responses and DIAMETER authentication procedures involved in the IMS signaling as well as SigComp compression benefits.

- We investigate the effects of tightly and loosely coupled interworking architectures on the SIP-based IMS registration procedure. We also provide insights into the delay efficiency of WiMax-3G interworking architectures.

The remainder of this paper is organized as follows: Section 2 describes IMS registration procedure. Section 3 presents our proposed delay analysis model for SIP-based IMS registration as well as our link layer analysis of SIP messages. The effects of WiMax-3G interworking architectures on IMS signaling delay are also explored in Section 3. Numerical results are presented in Section 4 and conclusions are given in Section 5.

\section{The IMS Registration Procedure}

In order to provide background for IMS registration analysis, we briefly describe the IMS registration procedure [1], [3]. For brevity, we limit our discussion to relevant IMS registration steps (see [1], [3] for further details). The IMS registration step numbers correspond to the numbers in Figure 1.

The IMS registration is a mandatory procedure in which the IMS user requests authorization to use the IMS services and consists of the following steps (Figure 1): 1)

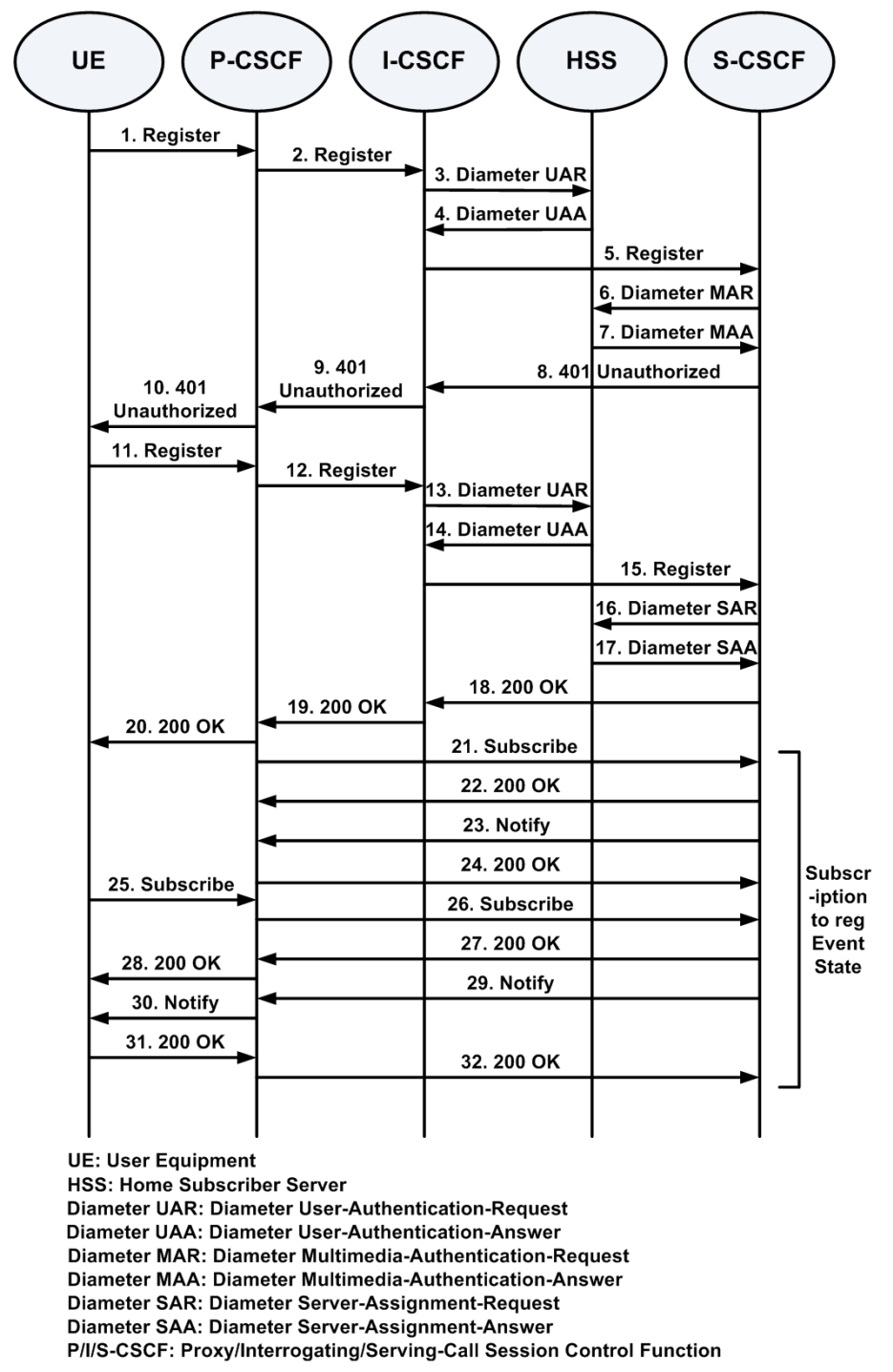

Figure 1. The IMS registration process [1], [3].

The IMS registration begins with a user equipment (UE) (a generic term for either SN or CN) SIP REGISTER request sent to the P-CSCF (proxy-call session control function). 2) The P-CSCF forwards the SIP REGISTER request to the I-CSCF (interrogating-call session control function) in the user's home network. 3) The I-CSCF sends a Diameter User-Authentication-Request (UAR) to the home subscriber server (HSS) for authorization and determination of S-CSCF (serving-call session control function) already allocated to the user. 4) The HSS authorizes the user and responds with a Diameter User-AuthenticationAnswer (UAA). 5) The I-CSCF forwards the SIP REGISTER request to the S-CSCF. 6) The S-CSCF sends a Diameter Multimedia-Authentication-Request (MAR) message to 
the HSS for downloading user authentication data. The SCSCF also stores its uniform resource indicator (URI) in the HSS. 7) The HSS responds with a Diameter MultimediaAuthentication-Answer (MAA) message with one or more authentication vectors. 8) - 10) The S-CSCF creates a SIP 401 Unauthorized response with a challenge question that the UESN must answer. 11), 12), \& 15) The UE responds with the answer to the challenge question in a new SIP REGISTER request. 16) If authentication is successful, the S-CSCF sends a Diameter Server-Assignment-Request (SAR) to inform the HSS that the user is registered and the HSS can download the user profile. 17) The HSS replies with a Diameter Server-Assignment-Answer (SAA). 18) 20) The S-CSCF sends a $200 \mathrm{OK}$ message to inform the user of successful registration. The subscription to a reg event state provides the user with his/her IMS network registration status. 25) \& 26) The UE sends a reg event SUBSCRIBE request to the $\mathrm{P}-\mathrm{CSCF}$, which then proxies the request to the S-CSCF. 27) \& 28) The S-CSCF sends a 200 OK after accepting the reg event subscription. 29) \& 30) The S-CSCF also sends a NOTIFY request containing registration information in extensible markup language (XML) format. 31) \& 32) The UE finishes the subscription to the reg event state process by sending a $200 \mathrm{OK}$ message. Note that steps 21) - 24) represent the reg event state subscription process for the P-CSCF and follow the same procedure as in steps 25) - 32).

\section{Delay Analysis Model for the IMS Registra- tion Procedure}

In this Section, we briefly describe the delay analysis model extension for the IMS registration procedure (see [10] for further details). The IMS registration signaling delay is comprised of three components, i.e., transmission delay, processing delay, and queueing delay.

$$
\bar{D}=D_{t}+D_{p}+D_{q}
$$

where $\bar{D}, D_{t}, D_{p}$, and $D_{q}$ denote the total average IMS signaling delay, transmission delay, processing delay, and queueing delay, respectively.

\subsection{Transmission Delay}

The transmission delay is the delay incurred due to signaling message transmission and is dependent on message size and channel bandwidth. The IMS registration procedure, including subscription to the reg event state, consists of eight message exchanges between the UE and the PCSCF server of the IMS network (Message No. 1, 10, 11, 20, 25, 28, 30, and 31 in Figure 1). 3G networks use RLP (an automatic repeat request (ARQ) MAC layer wireless interface protocol) to improve frame error rate (FER) performance, whereas WiMax networks do not use RLP due to much higher available bandwidth [2]. The IMS registration transmission delay in $3 \mathrm{G}$ networks $D_{t-i m s r e g-3 g}$ is:

$$
D_{t-i m s r e g-3 g}=8 \times D_{T C P w i t h R L P}
$$

The IMS registration transmission delay in WiMax networks $D_{t-i m s r e g-w i m a x}$ is:

$$
D_{t-i m s r e g-w i m a x}=8 \times D_{T C P n o R L P}
$$

where $D_{T C P \text { withRLP }}$ and $D_{T C P n o R L P}$ denote the average delay for successful TCP segment transmission with and without RLP, respectively, and 8 denotes the number of IMS registration signaling messages exchanged between the UE and the P-CSCF ([4] derives $D_{T C P \text { withRLP }}$ and $D_{\text {TCPnoRLP }}$ ).

\subsection{Processing Delay}

The processing delay is the delay incurred during packet encapsulation and decapsulation at the network layer. The IMS registration processing delay $D_{p-i m s r e g}$ is:

$$
\begin{aligned}
D_{p-i m s r e g}= & 4 d_{p-s n}+10 d_{p-p c s c f}+6 d_{p-i c s c f} \\
& +4 d_{p-h s s}+8 d_{p-s c s c f}
\end{aligned}
$$

where integers denote the number of IMS registration signaling messages processed at respective nodes and $d_{p-s n}$, $d_{p-p c s c f}, d_{p-i c s c f}, d_{p-h s s}$, and $d_{p-s c s c f}$ denote the unit packet processing delay at the SN, P-CSCF, I-CSCF, HSS, and S-CSCF, respectively (as calculated by [10]).

\subsection{Queueing Delay}

The queueing delay is the delay incurred due to packet queueing at network nodes. We assume $\mathrm{M} / \mathrm{M} / 1$ queues for the network nodes and a Poisson process for signaling arrival rate. The IMS registration queueing delay $D_{q-i m s r e g}$ is:

$$
\begin{aligned}
D_{q-i m s r e g}= & 4 E\left[w_{s n}\right]+10 E\left[w_{p c s c f}\right]+6 E\left[w_{i c s c f}\right] \\
& +4 E\left[w_{h s s}\right]+8 E\left[w_{s c s c f}\right]
\end{aligned}
$$

where integers denote the number of IMS registration signaling messages received at respective nodes and $E\left[w_{s n}\right]$, $E\left[w_{p c s c f}\right], E\left[w_{i c s c f}\right], E\left[w_{h s s}\right]$, and $E\left[w_{s c s c f}\right]$ denote the expected unit packet queueing delay at the SN, P-CSCF, I-CSCF, HSS, and S-CSCF, respectively (as calculated by [10]). 


\subsection{Total Delay}

The total IMS registration delay for a $3 \mathrm{G}$ network is:

$$
\begin{aligned}
\bar{D}_{\text {imsreg-3g }}= & D_{t-i m s r e g-3 g}+D_{p-i m s r e g} \\
& +D_{q-i m s r e g}
\end{aligned}
$$

The total IMS registration delay for a WiMax network is:

$$
\begin{aligned}
\bar{D}_{\text {imsreg-wimax }}= & D_{t-\text {-imsreg-wimax }}+D_{p-\text { imsreg }} \\
& +D_{q-\text { imsreg }}
\end{aligned}
$$

\subsection{SIP Message Application and Link Layer Analysis in WiMax and 3G Net- works}

This subsection analyzes the application layer SIP message sizes and associated link layer frames after SigCompbased compression. SigComp can reduce SIP message sizes by as much as $88 \%$ with negligible compression and decompression overhead. In our analysis, we use compression rates of 55\% and $80 \%$ for initial SIP messages (such as REGISTER) and subsequent SIP messages (200 OK, SUBSCRIBE, NOTIFY, 401 UNAUTHORIZED), respectively [9], [12]. Using these compression rates, the SIP message size for REGISTER is 225 bytes and all subsequent SIP messages are 100 bytes.

We calculate the number of frames per packet $K$ for different $3 \mathrm{G}$ and WiMax channel rates using a $3 \mathrm{G}$ transmission model [4] and its extension for WiMax. For the 3G network, we consider $19.2 \mathrm{kbps}$ and $128 \mathrm{kbps}$ channel rates and for the WiMax network, we consider 4 Mbps and 24 Mbps channel rates. For the $3 \mathrm{G}$ network, the RLP frame duration and inter-frame time $\tau$ is assumed to be $20 \mathrm{~ms}$ [4]. The WiMax frame duration and inter-frame time is assumed to be $2.5 \mathrm{~ms}$ and is independent of the channel rate [6]. For the $19.2 \mathrm{kbps} 3 \mathrm{G}$ network channel, each frame consists of $19.2 \times 10^{3} \times 20 \times 10^{-3} \times \frac{1}{8}=48$ bytes. For the SIP REGISTER message, $\mathrm{K}=\left\lceil\frac{225}{48}\right\rceil=5$. Following the same methodology, Table 1 shows the $K$ values for all the relevant IMS registration messages.

\subsection{Interworking Architectures and the IMS Registration Delay}

In our analysis, we study two different interworking architectures: tightly and loosely coupled. For the tightly coupled system, we consider the Tightly Coupled WiMaxCellular (TCWC) interworking architecture, specifically focusing on the WiMax-3G interworking portion of the Tightly Coupled Satellite-Cellular-WiMax-WLAN (TCSCW2) interworking architecture [11]. For the loosely coupled system, we consider the Loosely Coupled WiMaxCellular (LCWC) interworking architecture, specifically
Table 1. Number of frames per packet $K$ for various $3 G$ (19.2 and $128 \mathrm{kpbs})$ and WiMax (4 and $24 \mathrm{Mbps}$ ) channel rates

\begin{tabular}{|c|c|c|}
\hline Channel Rate & SIP REGISTER & SIP 200 OK \\
\hline $19.2 \mathrm{kbps}$ & 5 & 3 \\
\hline $128 \mathrm{kbps}$ & 1 & 1 \\
\hline $4 \mathrm{Mbps}$ & 1 & 1 \\
\hline $24 \mathrm{Mbps}$ & 1 & 1 \\
\hline
\end{tabular}

focusing on the WiMax-3G interworking portion of the Loosely Coupled Satellite-Cellular-WiMax-WLAN (LCSCW2) interworking architecture [11]. In both systems, UE must register with IMS network before IMS session establishment.

Our delay analysis is equally valid for all WiMax-3G interworking architectures supporting IMS services. Different architectures cause the IMS registration signaling messages (sent from the IMS terminal (UE) to the first point of contact with the IMS network) to flow between different architecture specific nodes. More specifically, for different architectures, different network nodes will be along the path between the UE (SN or CN) and the P-CSCF. To aggregate total delay, these differences require specific modeling of the network nodes involved. For the TCWC architecture, the total delay from the SN to the P-CSCF in a 3G network includes the delays incurred at the base station controller (BSC), radio network controller (RNC), SGSN (serving GPRS support node)/PCF (packet control function), and GGSN (gateway GPRS support node)/PDSN (packet data serving node). It should be noted that the SIP messages are transmitted on wireless links from the SN to the BSC while the GGSN will be hard-wired to the P-CSCF. Similarly, delays incurred at the packet data gateway (PDG), wireless access gateway (WAG), WiMax network controller (WNC), and WiMax base station controller (WBSC) should be added to the signaling delay from the P-CSCF to the $\mathrm{CN}$ in a WiMax network. For the LCWC architecture, delays incurred at the BSC, RNC, SGSN/PCF, and GGSN/PDSN constitute the additional delay from the $\mathrm{SN}$ to the P-CSCF in a $3 \mathrm{G}$ network. The delays incurred at the Internet, WAG, WNC, and WBSC constitute the incremental delay from the $\mathrm{P}-\mathrm{CSCF}$ to the $\mathrm{CN}$ in a WiMax network.

\section{Numerical Delay Analysis}

In this section, we present the numerical delay analysis for the SIP-based IMS registration procedure in WiMax-3G interworking architectures. We represent frame error probability as $p$, which can be obtained from the FER. The unit 


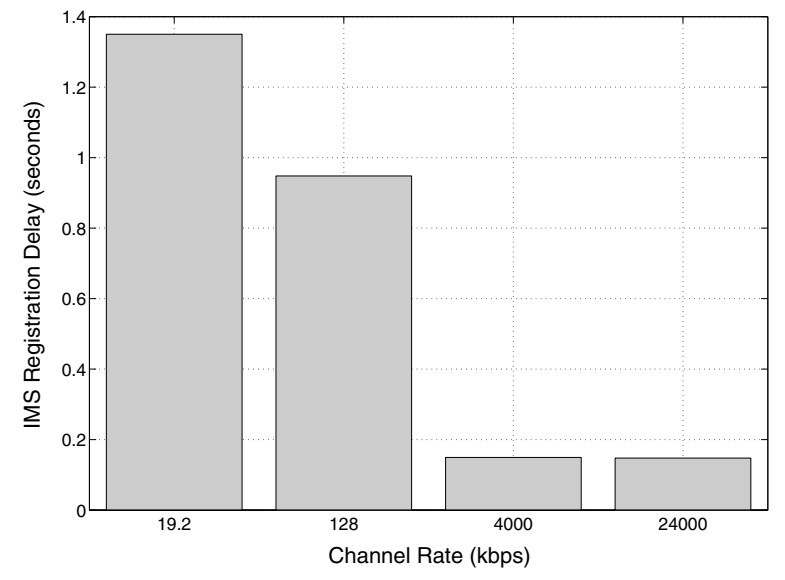

Figure 2. IMS registration signaling delay for various channel rates for a fixed signal arrival rate $\lambda$ and frame error probability $p$.

packet processing delay for SGSN/PCF, GGSN/PDSN, and the Internet is assumed to be $8 \times 10^{-3}$ seconds. The unit packet processing delay for the remainder of the network nodes is assumed to be $4 \times 10^{-3}$ seconds [7], [8], [10]. For queueing delay calculation, the service rate $\mu$ at all the network nodes is assumed to be 250 packets per second. The signaling and data traffic from other network sources constitute the background utilization at network nodes. Since the HSS must handle network traffic from different ANs, the HSS background utilization is assumed to be 0.7 , whereas for the remainder of the network nodes, the background utilization is assumed to be 0.4 . The background utilization for SGSN/PCF, GGSN/PDSN, and the Internet is assumed to be $0.5,0.5$, and 0.7, respectively. Further details about the selected parameters can be found in [10], [11].

The first experiment analyzes the IMS registration signaling delay for $3 \mathrm{G}$ network channel rates of $19.2 \mathrm{kbps}$ and $128 \mathrm{kbps}$ and WiMax network channel rates of $4 \mathrm{Mbps}$ and $24 \mathrm{Mbps}$. The frame error probability rate $p$ and the IMS signaling arrival rate $\lambda$ in packets per second are fixed at 0.02 and 9 , respectively.

Figure 2 shows IMS registration signaling delay in seconds versus varying channel rates. The figure shows that for $3 \mathrm{G}$ networks, the IMS registration signaling delay decreases with increased channel rate, while for the WiMax network, the delay remains nearly constant with increased channel rate. Furthermore, the delay for WiMax networks is considerably less than for $3 \mathrm{G}$ networks. These results are due to high WiMax channel rates, which reduce transmission delay effects.

The second experiment analyzes the effect of changing the IMS signaling arrival rate $\lambda$ in packets per second on

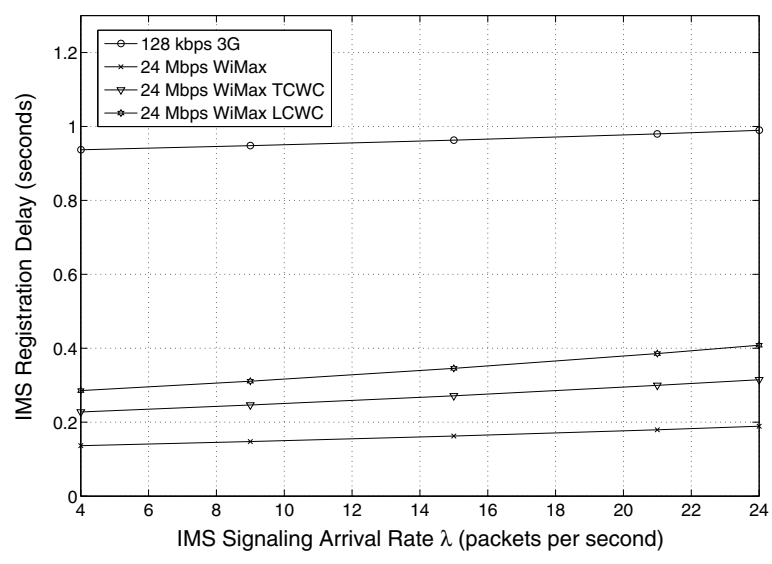

Figure 3. The effect of varying arrival rate $\lambda$ on the IMS registration signaling delay for 128 kbps 3G and 24 Mbps WiMax networks with a fixed frame error probability $p$.

the IMS registration signaling delay. The frame error probability $p$ is fixed at 0.02 . Results are calculated for arrival rates $\lambda$ of $4,9,15,21$, and 24 packets per second. The 3G and WiMax networks use $128 \mathrm{kbps}$ and $24 \mathrm{Mbps}$ channel rates, respectively. These results also analyze the effects of interworking architectures on the IMS registration signaling delay for different arrival rates.

Figure 3 shows IMS registration delay in seconds versus varying IMS signaling arrival rates for a $128 \mathrm{kbps} 3 \mathrm{G}$ network and 24 Mbps WiMax network for TCWC and LCWC architectures. The figure shows that the IMS registration signaling delay increases gradually with increasing arrival rate. This increase is in accordance with the queuing theory phenomenon where increased arrival rates result in increased network queue sizes and increased packet queuing time. Figure 3 also shows that the IMS registration signaling delay in the TCWC architecture is lower than in the LCWC architecture.

The third experiment analyzes the effect of frame error probability $p$ on the IMS registration signaling delay. The arrival rate $\lambda$ is fixed at 9 packets per second. We examine frame error probability $p$ values of $0.01,0.02,0.05,0.1$, and 0.2 for channel rates of $128 \mathrm{kbps}$ and $24 \mathrm{Mbps}$ for $3 \mathrm{G}$ and WiMax networks, respectively.

Figure 4 depicts the IMS registration signaling delay in seconds versus varying frame error probabilities for a $128 \mathrm{kbps} 3 \mathrm{G}$ network and a $24 \mathrm{Mbps}$ WiMax network for TCWC and LCWC architectures. The figure shows that the IMS registration signaling delay in $3 \mathrm{G}$ networks increases gradually while frame error probability increases, whereas frame error rate has negligible effect on the IMS registra- 


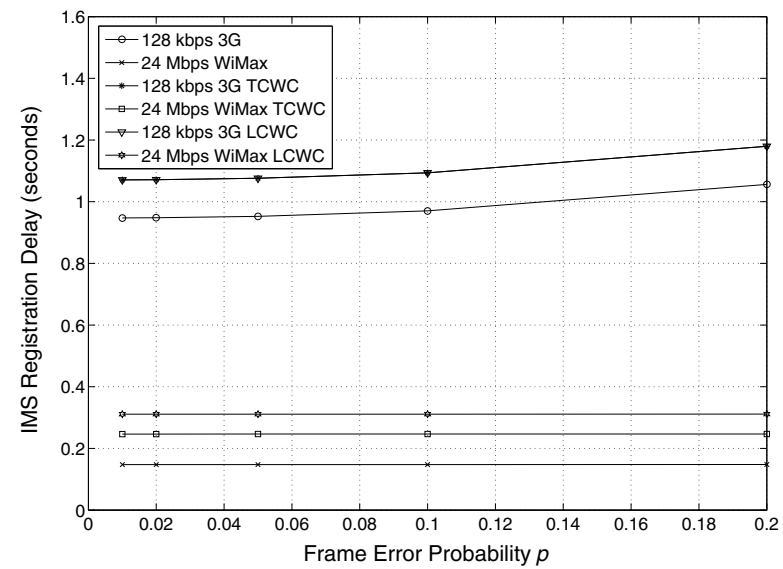

Figure 4. The effect of varying frame error probability $p$ on the IMS registration signaling delay for $128 \mathrm{kbps} 3 \mathrm{G}$ and $24 \mathrm{Mbps}$ WiMax networks with a fixed signaling arrival rate $\lambda$.

tion signaling delay for WiMax networks. The IMS registration delay for $3 \mathrm{G}$ networks is the same for both TCWC and LCWC interworking architectures due to identical additional network nodes in the path from the UE to the P-CSCF. However, for the WiMax network, the IMS registration delay for the TCWC architecture is less than the LCWC architecture due to different network nodes along the path from the UE to the P-CSCF.

\section{Conclusion}

In this paper, we analyzed the SIP-based IMS registration signaling delay in $3 \mathrm{G}$ and WiMax access networks. We also analyzed the effects of WiMax-3G interworking architectures on IMS signaling delay. The numerical data analysis reveals that the tightly coupled architectures have lower IMS signaling delays than the loosely coupled architectures. It can be concluded that a tightly coupled system is better suited for restricting the IMS signaling delays to affordable limits. However, tightly coupled architecture deployment requires more effort than loosely coupled architecture deployment, and hence a definite tradeoff exists between performance efficiency and implementation cost. Numerical data analysis indicates that the IMS registration signaling delay in WiMax networks is much less than the IMS registration signaling delay in $3 \mathrm{G}$ networks. These results encourage the deployment of WiMax-3G interworking architectures with IMS infrastructure support. Our future work focuses on transmission delay analytical model derivation for WiMax networks that closely follows the WiMax specifications, in addition to rigorous verification of our results (initial verification efforts have validated our results).

\section{Acknowledgment}

This work was partly supported by Bell Canada and Natural Sciences and Engineering Research Council of Canada (NSERC) under the supervision of Dr. Vincent Wong.

\section{References}

[1] 3GPP. Signalling flows for the IP multimedia call control based on Session Initiation Protocol (SIP) and Session Description Protocol (SDP); Stage 3 (Release 5). TS 24.228 (v5.15.0), September 2006.

[2] N. Banerjee, W. Wu, K. Basu, and S. Das. Analysis of SIP-based Mobility Management in 4G Wireless Networks. Elsevier Computer Communications, 27(8):697-707, May 2004.

[3] G. Camarillo and M.-A. Garcia-Martin. The 3G IP Multimedia Subsystem (IMS): Merging the Internet and the Cellular Worlds. John Wiley and Sons, 2004.

[4] S. Das, E.Lee, K. Basu, and S. Sen. Performance Optimization of VoIP Calls over Wireless Links using H.323 Protocol. IEEE Transactions on Computers, 52(6):742-752, June 2003.

[5] H. Fathi, S. Chakraborty, and R. Prasad. Optimization of SIP Session Setup Delay for VoIP in 3G Wireless Networks. IEEE Transactions on Mobile Computing, 5(9):1121-1132, September 2006.

[6] C. Hoymann, K. Klagges, and M. Schinnenburg. Multihop Communication in Relay Enhanced IEEE 802.16 Networks. In Proc. of 17th Annual IEEE International Symposium on Personal, Indoor and Mobile Radio Communications (PIMRC), Helsinki, Finland, September 2006.

[7] C. Liu and C. Zhou. An Improved Interworking Architecture for UMTS-WLAN Tight Coupling. In Proc. of IEEE Wireless Communications and Networking Conference (WCNC'05), Atlanta, Georgia, March 2005.

[8] C. Liu and C. Zhou. HCRAS: A Novel Hybrid Internetworking Architecture between WLAN and UMTS Cellular Networks. In Proc. of IEEE Consumer Communications and Networking Conference (CCNC'05), Las Vegas, Nevada, January 2005.

[9] M. Melnyk and A. Jukan. On Signaling Efficiency for Call Setup in all-IP Wireless Networks. In Proc. of IEEE International Conference on Communications (ICC'06), Istanbul, Turkey, June 2006.

[10] A. Munir. Analysis of SIP-based IMS Session Establishment Signaling for WiMax-3G Networks. In Proc. of IEEE International Conference on Networking and Services (ICNS'08), Gosier, Guadeloupe, March 2008.

[11] A. Munir and V. Wong. Interworking Architectures for IP Multimedia Subsystems. ACM/Springer Journal on Mobile Networks and Applications, 12(5):296-308, December 2007.

[12] R. Price, C. Bormann, J. Christoffersson, H. Hannu, Z. Liu, and J. Rosenberg. Signaling Compression (SigComp). RFC 3320, January 2003. 